\title{
A discussion of bias in magnetotelluric responses
}

\author{
M. Cristina Pomposiello ${ }^{1}$, John R. Booker ${ }^{2}$, and Alicia Favetto ${ }^{1}$
}

\begin{abstract}
Magnetic-field noise biases magnitudes of magnetotelluric response functions such as resistivity, impedance, and the induction vector (tipper). This is particularly severe in the socalled dead band between about 1 - and 10-s periods. The standard approach to remove these biases is to use a magnetic remote reference. In cases where this is unavailable or does not work because noise is correlated at the local and remote sites, it is possible to extract unbiased estimates using an inversion algorithm that predicts the magnitude from the phase. However, an important condition for this to be successful is that phase estimates are unbiased. Phase can be biased if parallel components of the electric and magnetic fields are correlated. This arises when the structure is $3 \mathrm{D}$ or when a $2 \mathrm{D}$ response is estimated in coordinates not parallel to strike. Improvements in electric field measurements make electric field references a viable option for estimating low-bias responses up to periods approaching $1000 \mathrm{~s}$.
\end{abstract}

\section{INTRODUCTION}

The standard way of removing bias caused by magnetic noise from magnetotelluric (MT) apparent resistivities is to apply the remote-reference method, in which magnetic components are measured simultaneously at two sites assumed to have correlated signals but uncorrelated noise (Gamble et al., 1979a, 1979b). However, a remote reference may fail to record the necessary data, may prove too noisy to produce reliable estimates, or may even produce biased estimates because of correlation between noise at the local and reference sites. It is important to determine which data are free of bias and can be used to determine strike or be included in an inversion for structure. Furthermore, the modern trend in MT data collection involves deploying many independent synchronized instruments. Initial processing to detect instrumental problems and provide feedback for deployment decisions may be more convenient in a single-site mode even when the ultimate processing will use multisite methods.

We have suggested using the rhoplus algorithm of Parker and Booker (1996) to detect biased resistivities (Pomposiello et al., 2002) and to predict their unbiased values. In this note, we confirm the technique by comparing remote-reference processing to using rhoplus with single-site processing. We demonstrate that it is useful with multidimensional MT responses even when strike is unknown and for induction vectors and diagonal impedance elements. Finally, we demonstrate that improvements in electric field measurements make electric field references a viable option.

\section{BIAS IN MT RESPONSES}

The MT impedance tensor $\mathbf{Z}$ is defined by a linear model of the complex frequency-domain relationship between horizontal electric $\mathbf{E}$ and horizontal magnetic $\mathbf{H}$ vector fields at the earth's surface:

$$
\mathbf{E}=\mathbf{Z H} .
$$

Post multiplying this equation by the complex-conjugate transpose of $\mathbf{H}$, denoted $\mathbf{H}^{*}$, and solving gives

$$
\mathbf{Z}=\mathbf{E} \mathbf{H}^{*}\left(\mathbf{H H}^{*}\right)^{-\mathbf{1}} .
$$

This is called the local $H$-reference estimate of $\mathbf{Z}$. Alternatively, one can multiply equation 1 by the inverse of $\mathbf{Z}$ :

$$
\mathbf{Z}^{-1} \mathbf{E}=\mathbf{H} .
$$

Postmultiplying this equation by the complex-conjugate transpose of $\mathbf{E}$, denoted $\mathbf{E}^{*}$, and solving gives

$$
\mathbf{Z}^{-1}=\mathbf{H E}^{*}\left(\mathbf{E E}^{*}\right)^{-1}
$$

The local $E$-reference impedance estimate is then

$$
\mathbf{Z}=\mathbf{E E}^{*}\left(H \mathbf{E}^{*}\right)^{-1} .
$$

The apparent resistivity is related to the magnitude of each element of $\mathbf{Z}$ by

Manuscript received by the Editor 3 March 2008; published online 26 June 2009

${ }^{1}$ Ciudad Universitaria, Instituto de Geocronolgía y Geología, Isotópica, CONICET, Pabellón INGEIS, Buenos Aires, Argentina. E-mail: cpomposi@ingeis uba.ar; favetto@ingeis.uba.ar.

${ }^{2}$ University of Washington, Department of Earth and Space Sciences, Seattle, Washington, U.S.A. E-mail: booker@ ess.washington.edu. (C) 2009 Society of Exploration Geophysicists. All rights reserved. 


$$
\rho_{i j}=0.2 \tau\left|Z_{i j}\right|^{2}
$$

where $\tau$ is the period. The value $\rho$ is measured in ohm-meters, and $\mathbf{Z}$ is measured in millivolts/kilometer/nanotesla $(\mathrm{mv} / \mathrm{km} / \mathrm{nT})$. The phase of each impedance element is

$$
\varphi_{i j}=\tan ^{-1}\left(\frac{\operatorname{Im}\left(Z_{i j}\right)}{\operatorname{Re}\left(Z_{i j}\right)}\right) .
$$

Only the off-diagonal terms $\rho_{x y}, \varphi_{x y}, \rho_{y x}$, and $\varphi_{y x}$ are referred to as the MT responses.

For practical computation, the elements of the matrices $\mathbf{E} \mathbf{H}^{*}$ and HH* must be cross-spectral estimates (Sims et al., 1971). When noise is present in the electric and magnetic channels, estimating the local $H$-reference impedance elements involves computing terms of the form

$$
\begin{aligned}
Z_{x y}= & \frac{\overline{\left(E_{x}+n_{e x}\right)\left(H_{y}+n_{h y}\right)^{*}\left(H_{x}+n_{h x}\right)\left(H_{x}+n_{h x}\right)^{*}}}{\left(E_{x}+n_{e x}\right)\left(H_{x}+n_{h x}\right)^{*}\left(H_{x}+n_{h x}\right)\left(H_{y}+n_{h y}\right)^{*}} \\
& -\overline{\left.\left(H_{x}+n_{h x}\right)\left(H_{x}+n_{h x}\right)^{*}\left(H_{y}+n_{h y}\right)\left(H_{y}+n_{h y}\right)^{*}\right)\left(H_{y}+n_{h y}\right)^{*}\left(H_{y}+n_{h y}\right)\left(H_{x}+n_{h x}\right)^{*}}
\end{aligned}
$$

where $\overline{A B^{*}}$ implies suitable averaging to produce a cross-spectral estimate and $n_{e x}, n_{e y}, n_{h x}$, and $n_{h y}$ are the noise in the orthogonal components of the electric and magnetic channels. If the noise components are uncorrelated with the signals and with each other, this becomes

$$
Z_{x y}=\frac{\overline{E_{x} H_{y}^{*}}\left(\left|\bar{H}_{x}\right|^{2}+\left|\bar{n}_{h x}\right|^{2}\right)-\overline{E_{x} H_{x}^{*} H_{x} H_{y}^{*}}}{\left(\left|\bar{H}_{x}\right|^{2}+\left|\bar{n}_{h x}\right|^{2}\right)\left(\left|\bar{H}_{y}\right|^{2}+\left|\bar{n}_{h y}\right|^{2}\right)-\left|\bar{H}_{x} H_{y}^{*}\right|^{2}},
$$

where $|\bar{A}|^{2} \equiv \overline{A A^{*}}$ is a power spectral estimate. The local $E$-reference estimate is

$$
Z_{x y}=\frac{\left(\left|\bar{E}_{x}\right|^{2}+\left|\bar{n}_{e x}\right|^{2}\right) \overline{H_{x} E_{y}^{*}}-\overline{E_{x} E_{y}^{*} E_{x} H_{y}^{*}}}{\overline{H_{y} E_{x}^{*} H_{x} E_{y}^{*}}-\overline{H_{y} E_{y}^{*} H_{x} E_{x}^{*}}} .
$$

Power spectral estimates are real and positive. The denominator of equation 3 is also real and positive because

$$
\left|\overline{H_{x} H_{y}^{*}}\right|^{2} \leq\left|\bar{H}_{x}\right|^{2}\left|\bar{H}_{y}\right|^{2} .
$$

The denominator of equation 3 obviously increases with magnetic-noise power at least as fast as the noise term in the numerator of equation 3 . Thus, the magnitude of the local $H$-reference estimates of $Z_{x y}$ and $\rho_{x y}$ always decrease with increasing magnetic noise and are said to be downward biased. Noise powers do not enter the denominator of equation 4 because it involves only cross-spectra of different field components. Thus, the magnitude of the local $E$-reference estimates of $Z_{x y}$ and $\rho_{x y}$ always increase with increasing electric noise and are upward biased.

The local $H$-reference estimate is preferable when the signal-tonoise ratio $(\mathrm{S} / \mathrm{N})$ of $\mathbf{H}$ is higher than $\mathbf{E}$. The local $E$-reference estimate is preferable when the $\mathrm{S} / \mathrm{N}$ of $\mathbf{E}$ is higher than $\mathbf{H}$. Historically, the former has been assumed true and local $E$-reference estimates are rarely computed routinely. Equivalent results hold for the other elements. This generalizes the 1D discussion of Sims et al. (1971).

The local $H$-reference estimate of the tangent of the phase of the $Z_{x y}$ is

$$
\begin{aligned}
\tan \varphi_{x y}= & \frac{\operatorname{Im} Z_{x y}}{\operatorname{Re} Z_{x y}} \\
= & \frac{\left(\left|\bar{H}_{x}\right|^{2}+\left|\bar{n}_{h x}\right|^{2}\right) \operatorname{Im}\left(\overline{E_{x} H_{y}^{*}}\right)-\operatorname{Im}\left(\overline{E_{x} H_{x}^{*} H_{x} H_{y}^{*}}\right)}{\left(\left|\bar{H}_{x}\right|^{2}+\left|\bar{n}_{h x}\right|^{2}\right) \operatorname{Re}\left(\overline{E_{x} H_{y}^{*}}\right)-\operatorname{Re}\left(\overline{E_{x} H_{x}^{*} H_{x} H_{y}^{*}}\right)} .
\end{aligned}
$$

Because the denominator of equation 3 is real, it cancels out of expression 5. Thus, the main source of bias in the impedance magnitude does not affect the phase. However, it is not true, as is sometimes stated, that phase is immune to bias. The remaining noise terms cancel out of expression 5 only if the conditions

$$
\overline{H_{x} H_{y}^{*}}=0
$$

or

$$
\overline{E_{x} H_{x}^{*}}=0
$$

are satisfied, i.e., the orthogonal components of $\mathbf{H}$ or the parallel components of $\mathbf{E}$ and $\mathbf{H}$ are uncorrelated. Three-dimensional structure or estimate of a $2 \mathrm{D} \mathbf{Z}$ in nonstrike coordinates can cause equation 7 to fail, and a strong 3D structure can generate anomalous magnetic fields for which equation 6 fails, even for completely uncorrelated external source-field polarizations. The direction of the bias may be up or down, and the diagonal element phases $\varphi_{x x}$ and $\varphi_{y y}$ are more susceptible to phase bias than $\varphi_{x y}$ and $\varphi_{y x}$. This is because equation 7 is replaced by a cross-spectrum between orthogonal components of $\mathbf{E}$ and $\mathbf{H}$, which always are large. The expression for local $E$-reference phase is more complicated than expression 5 because the denominator is complex and no longer cancels out. However, the electric-field noise power enters in essentially the same way as for the local $H$-reference.

The induction vector $\mathbf{T}_{z}$ is defined by the complex frequency-domain relationship

$$
H_{z}=\mathbf{T}_{z}^{t} \mathbf{H},
$$

where $H_{z}$ is the vertical component of the magnetic field and the superscript $t$ implies the vector transpose. Although not an MT response, it is commonly estimated at the same time. Postmultiplying this equation by $\mathbf{H}^{*}$ and solving gives

$$
\mathbf{T}_{z}^{t}=H_{z} \mathbf{H}^{*}\left(\mathbf{H H}^{*}\right)^{-\mathbf{1}} .
$$

If there is uncorrelated noise in all the input channels, the $x$-component of the induction vector becomes

$$
T_{z x}=\frac{\overline{H_{z} H_{x}^{*}}\left(\left|\bar{H}_{y}\right|^{2}+\left|\bar{n}_{h y}\right|^{2}\right)-\overline{H_{z} H_{y}^{*} H_{y} H_{x}^{*}}}{\left(\left|\bar{H}_{x}\right|^{2}+\left|\bar{n}_{h x}\right|^{2}\right)\left(\left|\bar{H}_{y}\right|^{2}+\left|\bar{n}_{h y}\right|^{2}\right)-\left|\bar{H}_{x} H_{y}^{*}\right|^{2}} .
$$

The component $T_{z y}$ is obtained by interchanging the $x$ and $y$ subscripts. The correspondence with equation 3 is obvious. If the condition in equation 6 holds, the magnitude of $T_{z x}$ will be biased downward by the same factor as $Z_{y x}$. More generally, the magnitude of $T_{z}$ is biased downward, and its phase is susceptible to significant bias un- 
less equation 6 holds because the cross-spectra of $H_{z}$ with the components of $\mathbf{H}$ are not likely to vanish.

The standard remote-reference technique involves replacing the conjugated magnetic field $\mathbf{H}^{*}$ in the development above with a transposed conjugated horizontal magnetic field $\mathbf{R}^{*}$ measured at a remote site. The remote $H$-reference estimate is

$$
\mathbf{Z}^{R}=\mathbf{E} \mathbf{R}^{*}\left(\mathbf{H} \mathbf{R}^{*}\right)^{-\mathbf{1}} .
$$

Terms in the local reference development, such as $\left|\bar{n}_{h x}\right|^{2} \rightarrow \overline{n_{h x} n_{r x}^{*}}$, vanish if the local and remote noise sources are uncorrelated. Then

$$
Z_{x y}^{R}=\frac{\overline{E_{x} R_{y}^{*} H_{x} R_{x}^{*}}-\overline{E_{x} R_{x}^{*} H_{x} R_{y}^{*}}}{\overline{H_{x} R_{x}^{*} H_{y} R_{y}^{*}}-\overline{H_{x} R_{y}^{*} H_{y} R_{x}^{*}}}
$$

no longer involves noise powers and hence its magnitude and phase are unbiased. Similar remote-reference estimates can be defined for the other elements of $\mathbf{Z}$, for $\mathbf{T}_{\mathrm{z}}$, and for a remote $E$-field reference. The remote-reference estimates may be poor estimates if the local and remote signals are poorly correlated.

\section{RHOPLUS}

The rhoplus algorithm solves the inverse problem of finding the 1D MT responses that fit a set of apparent-resistivity and impedancephase data best in the chi-squared misfit sense. Because the algorithm can invert resistivity $\rho$ or phase $\varphi$ independently, it is possible to use the algorithm to predict one from the other in a physically consistent way. This result would be of little practical importance if multidimensional models had $\rho$ - $\varphi$ relations substantially different than one dimension. However, Weidelt and Kaikonnen (1994) prove that the MT responses of 2D models with the electric field polarized perpendicular to strike (TM mode or $B$-polarization) always have a 1D $\rho-\varphi$ relation. Furthermore, 2D models with the electric field polarized parallel to strike (TE mode or $E$-polarization) have $1 \mathrm{D} \rho$ - $\varphi$ relations except in rare circumstances. Three-dimensional situations can have $\rho-\varphi$ relations that differ substantially from 1D situations. However, if three-dimensionality is local distortion of the electric field in a regional $2 \mathrm{D}$ structure and $\mathbf{Z}$ is in regional strike coordinates, all of its elements have 2D $\rho$ - $\varphi$ relations (see Smith, 1995 and references there) and will also almost always have $1 \mathrm{D} \rho-\varphi$ relations

If $\varphi$ is unbiased, one can detect bias in $\rho$ by predicting it from $\varphi$. However, a phase inversion contains an arbitrary constant. Downward bias in $\rho$ commonly is serious only in the so-called dead band $(1-10 \mathrm{~s})$ in which natural sources are weak and cultural noise may be strong. Because data normally are collected over a band much wider than the dead band, one usually has data at either longer or shorter periods that can be assumed reasonably unbiased. One can set the unknown constant by specifying $\rho$ at one period or including in the inversion $\rho$ within a band judged to have insignificant bias.

\section{FIELD EXAMPLES}

\section{Field sites and instrumentation}

We present data from two sites in Argentina. The first, tuc104 (27.129 $9^{\circ}$ south, $65.358^{\circ}$ west), is from Pomposiello et al. (2002). This site is in the western part of the Tucumán plain, a semirural area with nearby towns and potential sources of cultural noise such as light industrial plants, electric fences, water wells, and pipelines with cathodic protection. The data were collected with an EMI MT-24 system recording at $3.75 \mathrm{~Hz}$ and processed using a robust time-series code (Egbert and Booker, 1987). The electric dipoles were $100 \mathrm{~m}$ long and used liquid-filled $\mathrm{Pb}-\mathrm{PbCl}_{2}$ electrodes (Steve Park, personal communication, 1998). The second site, pam820 ( $31.754^{\circ}$ south, $62.288^{\circ}$ west), is near the boundary between Córdoba and Santa Fe provinces. This is a rural area with large farms and no significant urbanization within $50 \mathrm{~km}$.

The data were collected in September 2003 with a Narod Geophysics NIMS long-period MT system recording at $4 \mathrm{~Hz}$ and processed using the same robust code. The electric dipoles were also $100 \mathrm{~m}$ long but used $\mathrm{Pb}-\mathrm{PbCl}_{2}$ electrodes employing the design principles of Petiau (2000). The NIMS uses a fluxgate magnetic sensor that has significantly higher noise in the dead band than the induction coil magnetometer used by the MT- 24 .

\section{Prediction of unbiased responses using rhoplus}

Figure 1 shows the local and remote $H$-reference MT responses in measurement coordinates for tuc104. The single-site processing uses 13 hours of data. The remote-reference site is $11 \mathrm{~km}$ away, and the data overlap for 10 hours. The local reference $\rho_{y x}$ are biased up to an order of magnitude downward relative to the remote-reference estimates at periods shorter than $10 \mathrm{~s}$ (Figure 1a). In contrast, the local reference $\rho_{x y}$ have very little bias relative to the remote-reference estimates (Figure 1b). The phase estimates for both polarizations are almost identical for the local and remote references. Thus, the localreference phases are not biased.

Let normalized rms misfit be the square root of the chi-squared misfit statistic normalized by its value at $95 \%$ probability. Then compatibility with a $1 \mathrm{D}$ response requires that normalized rms of a rhoplus inversion be less than one. The rhoplus inversion of the local $H$-reference $\varphi_{y x}$ from 1.3 to $81 \mathrm{~s}$ has a normalized rms of 0.75 and is compatible with a $1 \mathrm{D} \varphi$ relation.

To predict resistivities, we need some resistivity information. We can choose any individual $\rho$ datum at $20 \mathrm{~s}$ or longer and get essentially the same result without altering the misfit to the phase. The responses, shown as squares in Figure 1a, include all five local $H$-ref $\rho_{y x}$ in the band from 20 to $81 \mathrm{~s}$. Although the normalized rms of 1.08 is slightly higher than for phase alone, the predicted resistivities are in excellent agreement with the remote-reference values. This demonstrates our fundamental point that unbiased resistivities can be predicted reliably from single-site data using rhoplus.

The normalized rms fit to $\varphi_{x y}$ is 1.15 , so this polarization is not strictly compatible with a $1 \mathrm{D} \varphi$ relation. The predicted $\rho_{x y}$, again shown as open squares in Figure 1b, are close to, but fall consistently above, the remote-reference estimates in the dead band. It is tempting to conclude that the remote-reference resistivities are slightly downward biased. We can use rhoplus to test this hypothesis.

First, we note that increasing all phase-error estimates by $20 \%$ brings the normalized rms below 1.0. However, this has no effect on the predicted values of resistivity or the appearance of bias with respect to the remote-reference values. Next, the width of the error envelope varies considerably with period. Thus, rhoplus will preferentially weight the phase more heavily at periods shorter than $6 \mathrm{~s}$. To see whether optimistic short-period phase errors cause the bias, we whiten the data weighting by putting a $1.43^{\circ}$ floor under the phase errors. Except at the shortest period, this new data weighting has no effect on apparent bias. In fact, inverting the remote-reference phase data with or without an error floor (not shown) leads to the same appearance of bias. So optimistic phase errors are not responsible. 
Next, we turn the problem around and invert the remote $H$-reference $\rho_{x y}$ for $\varphi_{x y}$. The normalized rms for this inversion is 0.73 . Thus, the remote $H$-reference resistivities are compatible with a $1 \mathrm{D}$ relation. However, the predicted phases shown in Figure 1c lie well outside their two standard error envelopes at the short and long periods. If, however, we whiten the data weights with a $5 \%$ error
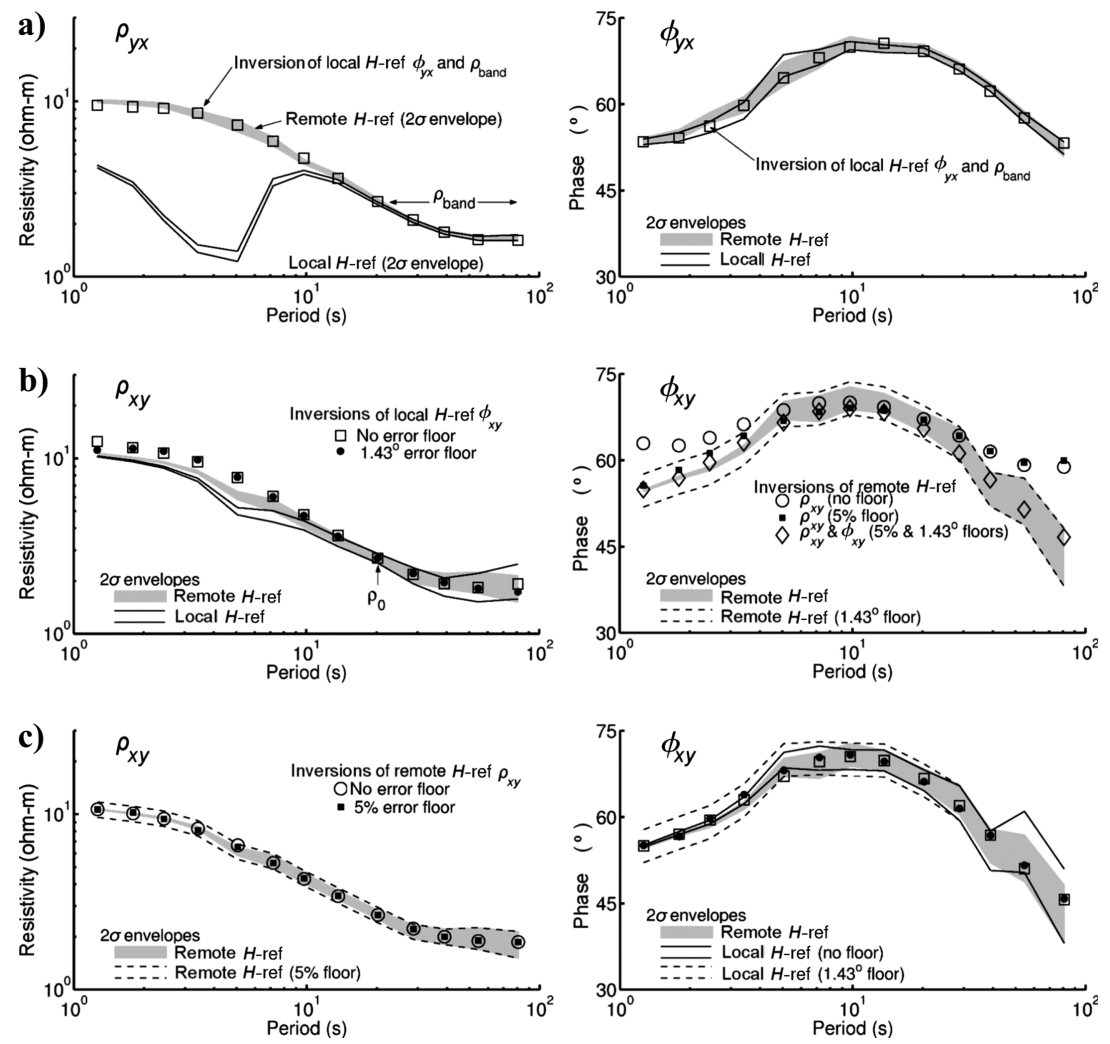

Figure 1. Local and remote-reference MT responses at site TUC104. The phases have been moved from the third to first quadrants. Only the \pm 2 estimated standard error envelopes are shown for the measurements. (a) Open squares are responses of the rhoplus inversion of $\varphi_{y x}$ and the five resistivities in $\rho_{\text {band }}$. (b) Open squares are responses of the rhoplus inversion of $\varphi_{x y}$ and the single resistivity $\rho_{0}$. The solid circles are the responses for the inversion the same data using a $1.43^{\circ}$ floor for the errors in $\varphi_{x y}$. The open diamonds are the responses of the inversion of both resistivity and phase with equivalent error floors. (c) Open circles are responses of the rhoplus inversion of $\rho_{x y}$. The solid squares are the responses for the inversion the same data using a $5 \%$ floor for the errors in $\rho_{x y}$.
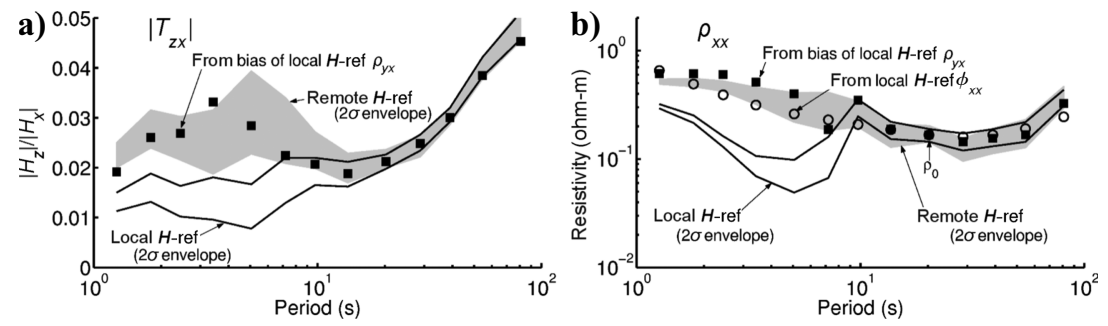

Figure 2. (a) Local and remote $H$-reference vertical-field responses at tuc104. The error envelopes are \pm 1 standard errors. The filled symbols are the vertical field magnitude from the bias in $\rho_{y x}$ estimated from local $H$-reference $\varphi_{y x}$. (b) Local and remote $H$-reference estimates of the apparent resistivity of $Z_{x x}$. The open circles are unbiased resistivities predicted by a rhoplus inversion of $\varphi_{x x}$ using $\rho_{x x}$ at $20 \mathrm{~s}$ to set the scale. The filled squares are the resistivities predicted by assuming that the bias in $\rho_{x x}$ is the same as the bias in $\rho_{y x}$. floor (equivalent to $1.43^{\circ}$ for phase), the resistivity responses change only subtly but the short-period phase discrepancy disappears. We conclude that the apparent short-period bias of the remote-reference is a consequence of underestimated apparent resistivity ror bars in the dead band.

invert $\rho$ and $\varphi$ with equivalent error floors (5\% and $1.43^{\circ}$ ), we get a normalized rms of 0.73 , and both responses are predicted within their error envelope across the entire bandwidth. This demonstrates a second important point: It is often useful to incorporate an error floor when error estimates vary widely to avoid overfitting data with error estimates that are too optimistic. Floors were not needed for the $y x$ polarization because the measured error estimates were already almost white.

Small bias in $\rho_{x y}$ implies that the noise power in $H_{y}$ is also small. The presence of strong bias in $\rho_{y x}$ requires that the noise power in $H_{x}$ be substantial. The noise power in $H_{x}$ can cancel out of equation 3 and leave $\rho_{x y}$ unbiased only if the condition in equation 6 is satisfied. This has several consequences. First, the magnitude of $T_{z x}$ should be biased by the same factor as $Z_{y x}$ (and hence the square root of $\rho_{y x}$ ), and $\rho_{x x}$ should be biased by the same factor as $\rho_{y x}$. We should thus be able to the use resistivity bias predicted by inverting the local $H$-reference $\varphi_{y x}$ to predict unbiased values of $T_{z x}$ and $\rho_{x x}$. Figure 2 shows that this works reasonably well.

The same reasoning leads to the conclusion that the second terms in the numerator and denominator of equation 5 and its analogs must be negligible and that bias caused by magnetic-field noise cancels out of the phase of all impedance elements. We can then predict $\rho_{x x}$ by inverting $\varphi_{x x}$. Figure $2 \mathrm{~b}$ shows that this predicts unbiased values in reasonable agreement with the remote-reference estimates.

The bias in $\rho_{y x}$ could be because of magnetic sensor noise if the $H_{x}$ source power is much smaller than the $H_{y}$ source power or if the $H_{x}$ sensor is malfunctioning. Lack of similar bias at the remote-reference site (not shown) almost certainly rules out a source-power effect. The fact that the same sensor and electronics combination never exhibited similar bias at other sites makes an instrument problem unlikely. We conclude that the noise is probably a highly polarized cultural source.

\section{Choice of coordinate system}

The $y x$ and $x y$ responses at tuc104 are similar, and $\rho_{x x}$ is an order of magnitude less than $\rho_{y x}$. This implies an almost 1D structure. Thus, structural dimensionality does not influence the choice of coordinate system in which to apply rhoplus. Because the bias is very different in the two polarizations, we do it in measurement coordinates. 
Figure 3 shows local $H$ - and $E$-reference MT responses from 2.56 to $2037 \mathrm{~s}$ at site pam820. This single-site processing uses 67 hours of data. Where they differ, all local $E$-reference $\rho$ estimates are above the local $H$-reference estimates as expected. The most obvious features of this comparison are the large downward bias of the local $H$-reference estimates of $\rho$ relative to the local $E$-reference estimates at short period and the close agreement between the two estimates of $\varphi$ at all periods. Although $\varphi$ for the two polarizations asymptote to one another at the shortest periods, there is a significant phase split that starts in the dead band and grows much larger at longer periods. These data are at least strongly two-dimensional.

Theoretical considerations suggest that the appropriate coordinate system in which to apply rhoplus to $2 \mathrm{D}$ data is aligned with strike. However, in the presence of significant bias, one cannot use impedance tensor decomposition (e.g., Smith, 1995) to determine strike. If there is also little geologic information to constrain strike definitively, it is useful to consider whether it matters if the coordinate system is strike oriented.

We first ascertain which data can be considered unbiased. Figure 4a shows the normalized rms to the local $H$-reference $\rho$ and $\varphi$ data in measurement coordinates as a function of excluding short-period resistivities. To even out the data weight over the full bandwidth, an error floor of $5 \%$ is applied to $\rho$, and its equivalent of $1.43^{\circ}$ has been applied to $\varphi$. After a sharp drop associated with inclusion of obviously biased $\rho$ data, the misfit levels out. By $30 \mathrm{~s}$, it is clear that the resistivities are no longer biased for either polarization. Figure $4 \mathrm{~b}$ shows the normalized rms misfit as a function of the rotation of the impedance tensor from measurement coordinates for inversions that exclude resistivities below 29 $\mathrm{s}$. The misfit at $0^{\circ}$ is to the $y x$ responses in measurement coordinates. Rotation of $H_{x}$ to $\pm 90^{\circ}$ corresponds to fitting the measured $x y$ polarization.

Also shown in Figure $4 \mathrm{~b}$ is the normalized rms of the predicted values of $\rho$ to the short-period remote $H$-reference $\rho$. It is essentially one or less over the entire range. Thus, it does not matter what coordinate system we use to predict unbiased resistivities from the local $H$-reference phase data.

Figure 5 compares the predicted responses at rotation steps of $15^{\circ}$ with the error envelopes of remote $H$-reference responses modified by the error floors. The remote-reference site was $24 \mathrm{~km}$ away and the data overlapped for 64 hours. The period dependence of the responses changes considerably with rotation angle, but the normalized rms for the inversion of the local-reference data is below one at almost all angles. This illustrates that it is unnecessary to get the strike approximately right for a $1 \mathrm{D} \rho-\varphi$ relation to be acceptable for the MT responses.

It is tempting to suppose that the misfit minimum around a rotation of $-45^{\circ}$ is somehow related to strike (Figure 4b). However, impedancetensor decomposition of the remote-reference impedance and geologic considerations give a north-south strike, with the $0^{\circ}$ rotation being the $\mathrm{TM}$ mode and the $90^{\circ}$ rotation the TE mode. The maximum rhoplus misfit at a rotation of $75^{\circ}\left(-15^{\circ}\right.$ rotation of the $x y$ response) does not correspond to even the most complicated responses (which are at $15^{\circ}-30^{\circ}$ ). We conclude that rhoplus misfits to MT responses tell little about strike. On the other hand, if $\mathbf{Z}$ is in strike coordinates and the model of a galvanic electric distortion of a regional $2 \mathrm{D}$ response holds, $Z_{x x}$ and $Z_{y y}$ should be real constants times $Z_{y x}$ and $Z_{x y}$ (e.g., Smith, 1995). A necessary condition for this distortion model to hold is that rhoplus fit the diagonal responses. A poor fit may be a sensitive measure of regional strike and model validity.

\section{Use of $E$-referenced phases}

Figure 6 focuses on the pam820 $x y$ responses of less than $120 \mathrm{~s}$ and compares the local-reference estimates to remote $H$ - and $E$-reference estimates. The remote-reference responses have higher error estimates than the local-reference estimates because of the noise in the reference channel. All four estimates of $\varphi_{x y}$ are essentially identical and thus unbiased. The remote $H$-reference responses have the largest error envelope. This is to be expected because the two-orders-of-magnitude downward bias of $\rho$ requires that the magneticsensor noise be one order of magnitude above the signal. Despite this, robust stacking of the large amount of data that can be collected with long-period systems using modern data storage estimates good
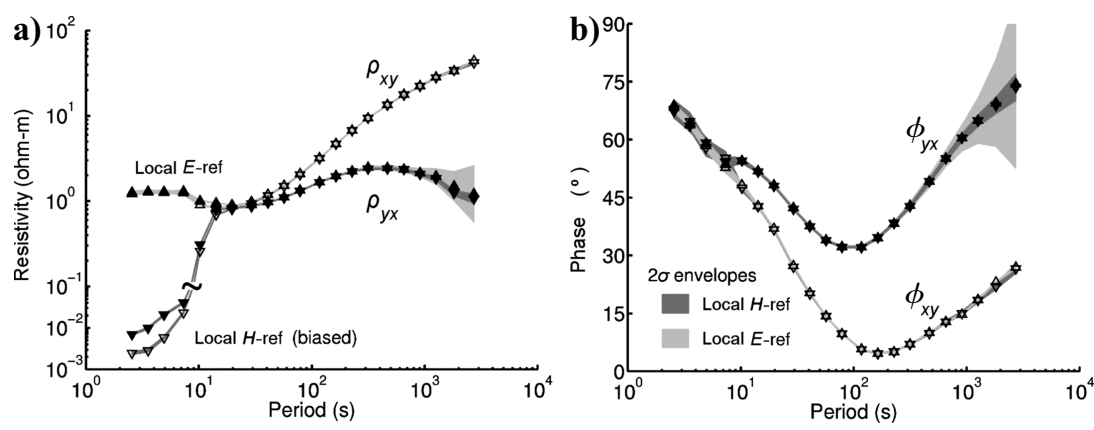

Figure 3. Local-reference MT responses at pam820. Downward triangles are $H$-reference estimates susceptible to downward bias; upward triangles are $E$-reference estimates susceptible to upward bias. When the data look like six-pointed stars, the two estimates are the same. Open symbols are the $x y$ polarization; filled symbols are the $y x$ polarization. The $y x$ phases have been moved from the third to first quadrants. Note the $\rho$ scale change at $10^{-2} \mathrm{ohm}-\mathrm{m}$. The error envelopes are \pm 2 estimated standard errors.
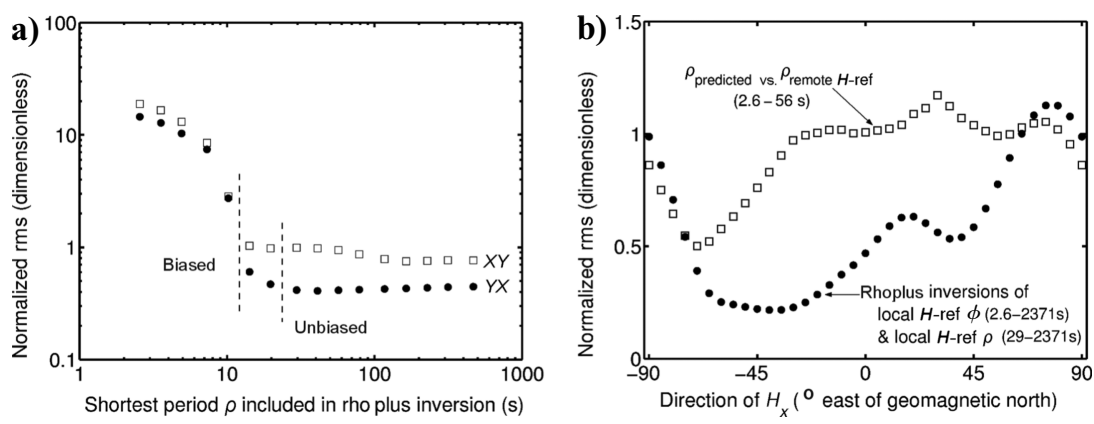

Figure 4. (a) Misfit of rhoplus inversions of pam820 responses with successive exclusion of resistivities at short period. (b) Misfit as a function of rotation angle. The filled circles are the local $H$-reference responses with $\rho$ at periods less than $29 \mathrm{~s}$ eliminated. The open squares are the misfit of $\rho$ predicted by the same inversion of the remote $H$-reference data in the bandwidth from 2.56 to $56 \mathrm{~s}$. Error floors of $5 \%$ in $\rho$ and its equivalent of $1.43^{\circ}$ in $\varphi$ were applied to all data before computing normalized rms in this figure. 
dead-band responses with equipment formerly considered unuseful in this band.

The local $E$-reference estimates of $\rho$ lie at the upper limit estimated using either remote reference. This indicates that the $\mathrm{S} / \mathrm{N}$ for $\mathbf{E}$ is high in the dead band and bias is small. The $\mathrm{S} / \mathrm{N}$ in $\mathbf{E}$ inevitably decreases with increasing period and decreasing $\rho$. This is reflected in the growing error estimates and upward bias of $\rho$ for the local $E$-reference $y x$ polarization beyond $1000 \mathrm{~s}$ (Figure 3). The local $E$-reference performs as well as the local $H$-reference at long periods for the higher-resistivity $x y$ polarization. This indicates local $E$-reference estimates using modern electrodes and electronics deserve more respect than they have received historically.

As before, an error floor $\pm 1.43^{\circ}$ (equivalent to $\pm 5 \%$ in $\rho$ ) equalizes the data weight across the 2.56-117-s bandwidth. The $\rho$ predicted from either the local $H$ - or $E$-reference phase data are almost identical and fall in the middle of the remote $H$-reference range. The predictions shown are for an inversion that includes three resistivities $\left(\rho_{\text {band }}\right)$; however, using any $\rho$ information above 30 s gives identical results.

The remote $E$-reference estimates in the dead band appear to be slightly biased relative to the remote $H$-reference and the responses predicted by rhoplus from the local $H$ - or $E$-reference responses. No combination of phase data weighting removes this appearance. The bias of the local $H$-reference $\rho$ estimates is caused almost certainly by internal magnetic sensor noise, which is extremely unlikely to be correlated between sites separated by $24 \mathrm{~km}$. Thus, bias of remote $H$-reference $\rho$ is unlikely. Any bias between the remote reference estimates must be in the $E$-reference and be caused by correlated noise in $\mathbf{E}$. It could be caused by a cultural source, but we think it is more likely an instrument problem. The prototype NIMS had a small step in the DC level of the $\mathbf{E}$ amplifiers when the GPS receiver turned on to resynchronize the data logger clock. Although no longer detect-
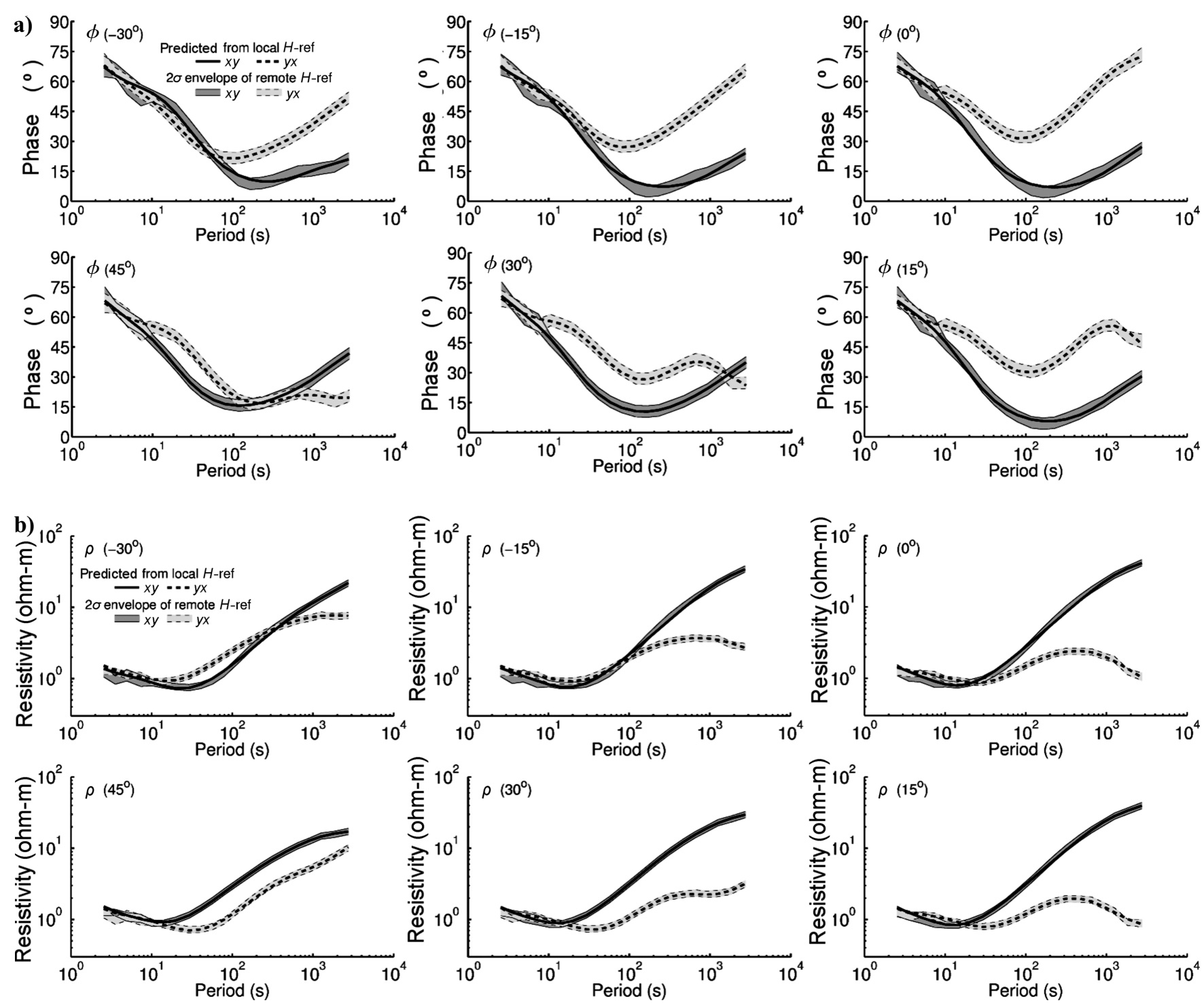

Figure 5. Responses of rhoplus inversion (heavy lines) compared to two standard-error envelopes of remote $H$-reference estimates (light lines) as a function of rotation of the pam 820 impedance tensor from measurement coordinates $\left(0^{\circ}\right)$. The solid lines correspond to rotations of the $y x$ polarization; the dashed lines correspond to rotations of the $x y$ polarization. 

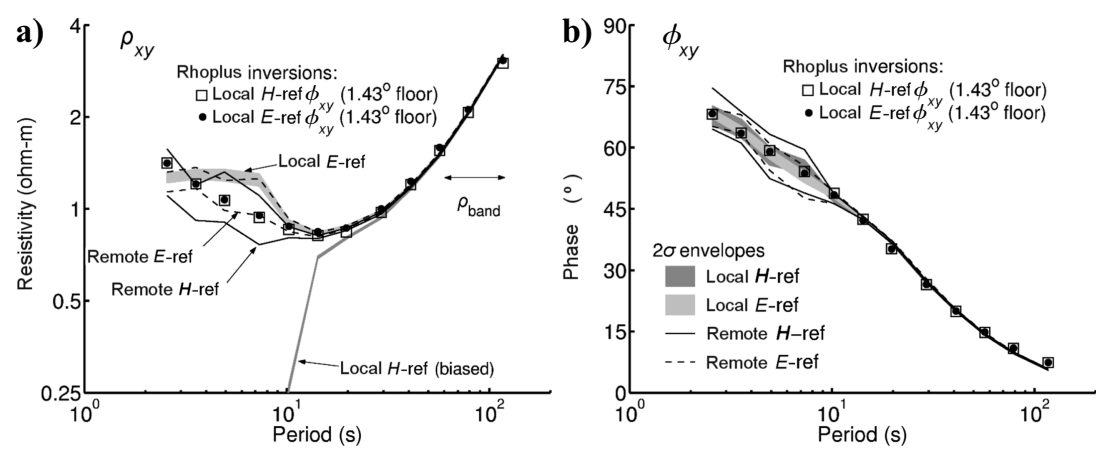

Figure 6. MT responses at pam820. Only the error envelopes of the measured data are shown. The symbols are the responses predicted by the rhoplus algorithm from the local $H$ - and $E$-reference phase data. The measured error envelope and inversion-response legends apply to both responses.

able by direct observation in our units, a residual effect may remain. The sharp leading edge of any such offset would introduce noise in the dead band. This would appear to be synchronized between units because the interval between clock resets in different units is the same within less than $1 \mathrm{~ms}$.

\section{CONCLUSIONS}

Successful prediction of unbiased resistivities from single-site data requires that the phase data be unbiased. At site tuc104, the small bias in one polarization requires that the orthogonal-source magnetic fields be nearly uncorrelated and leads immediately to a conclusion that the phases are unbiased. If this is a general property of natural dead-band sources, then phase bias can always be ignored unless strong three-dimensionality generates significant correlated orthogonal-horizontal magnetic fields. If the diagonal elements of $\mathbf{Z}$ are small, one also can be sure that the electric and magnetic fields in parallel directions are not highly correlated and phase bias of the offdiagonal elements is small even when source correlation is significant. This is true in the measurement coordinates at pam820, but not in all the rotations presented in Figures 4 and 5. Thus coherence between the orthogonal sources must be small at this site also. We have not found an example where the natural dead-band sources were highly correlated in orthogonal directions when averaged over many hours; this needs more investigation.

Successful prediction of unbiased resistivities from single-site data also requires that the $1 \mathrm{D} \rho$ - $\varphi$ relation be at least a good approximation. No general theory exists regarding the limits of applicability of this requirement, but experimental evidence suggests it is rather useful. In fact, a poor fit using rhoplus is a clear indication of serious 3D complications or instrument problems.

We do not suggest that rhoplus should be used in place of a remote reference. However, rhoplus predictions using single-site responses can be expected to do almost as well as a remote reference in remov- ing bias of the off-diagonal apparent resistivities when the phase is not contaminated seriously by noise or 3D effects. However, these predictions add no new information in a statistical sense. This means they add no additional degrees of freedom to a subsequent multidimensional, multisite inversion.

There are obvious practical uses of the $\rho$ values predicted by rhoplus. For instance, one can immediately conclude without the need for remotereference processing that the shallow structure at the sites presented in this paper is nearly one-dimensional because the rhoplus-predicted $\rho$ and $\varphi$ for both polarizations asymptote to one another. Also, one can construct quickly an unbiased resistivity pseudosection as one proceeds along a profile of independently recording systems without assembling files for multisite processing. Finally, in favorable circumstances, one can estimate unbiased induction vectors and diagonal impedance elements, permitting techniques such as tensor decomposition for strike determination when remote-reference estimates are unavailable.

\section{ACKNOWLEDGMENTS}

This research was supported by National Science Foundation (NSF) grants EAR99-09390 and EAR03-10113, and by Agencia Nacional de Promoción Científica y Tecnológica grant PICT 99: 07-06313. The MT systems were provided by the EMSOC Instrument Facility supported by NSF grants EAR02-36538 and EAR9616421.

\section{REFERENCES}

Egbert, G., and J. Booker, 1987, Robust estimation of geomagnetic transfer functions: Geophysical Journal of the Royal Astronomical Society, 87, 173-194.

Gamble, T. D., W. Goubau, and J. Clarke, 1979a, Magnetotellurics with a remote reference: Geophysics, 44, 53-68.

, 1979b, Error analysis for remote reference magnetotellurics: Geophysics, 44, 959-968

Parker, R., and J. Booker, 1996, Optimal one-dimensional inversion and bounding of magnetotelluric apparent resistivity and phase measurements: Physical Earth Planetary International, 98, 269-282.

Petiau, G., 2000, Second generation of lead-lead chloride electrodes for geophysical applications: Pure and Applied Geophysics, 157, 357-382.

Pomposiello, C., A. Favetto, C. Sainato, J. Booker, and S. Li, 2002, Imaging the sedimentary basin of the Tucumán plain in the northern Pampean Range Argentina: Journal of Applied Geophysics, 49, 47-58.

Sims, W. E., F. X. Bostick, Jr., and H. W. Smith, 1971, The estimation of magnetotelluric impedence tensor elements from measured data: Geophysics, 36, 938-942.

Smith, T., 1995, Understanding telluric distortion matrices: Geophysical Journal International, 122, 219-226.

Weidelt, P., and P. Kaikonnen, 1994, Local 1-D interpretation of magnetotelluric B-polarization impedances: Geophysical Journal International, 117, 733-748. 